PROCEEDINGS OF THE

AMERICAN MATHEMATICAL SOCIETY

Volume 134, Number 7, Pages 2049-2060

S 0002-9939(06)08208-6

Article electronically published on January 5, 2006

\title{
LÊ MODULES AND TRACES
}

\author{
DAVID B. MASSEY
}

(Communicated by Ronald A. Fintushel)

\begin{abstract}
We show how some of our recent results clarify the relationship between the Lê numbers and the cohomology of the Milnor fiber of a nonisolated hypersurface singularity. The Lê numbers are actually the ranks of the free Abelian groups - the Lê modules-appearing in a complex whose cohomology is that of the Milnor fiber. Moreover, the Milnor monodromy acts on the Lê module complex, and we describe the traces of these monodromy actions in terms of the topology of the critical locus.
\end{abstract}

\section{INTRODUCTION}

From 1988 to 1995, we developed the Lê numbers as a generalization to nonisolated affine hypersurface singularities of the Milnor number of an isolated hypersurface singularity; see 10. Since producing the Lê cycles and Lê numbers, we have wondered how to generalize their construction to the completely general case of a function $f: X \rightarrow \mathbb{C}$, where $X$ itself may be an arbitrarily singular space and $f$ has an arbitrary critical locus. Of course, what the "critical locus" of such an $f$ should mean is part of the problem.

Recently, in [11, we developed a general framework for producing "Lê-like" cycles, associated to such general $f$. We found that the correct generalization of the Lê cycles were a type of cycle where the coefficients were not integers, but were, in fact, modules. Moreover, these "enriched cycles" were graded. Similarly, the Lê numbers were replaced by graded modules; to obtain actual numbers, one had to take the Euler characteristics of these graded modules.

The main problem with [1] is that the setting is so general that it is difficult to translate the results into statements that are more down-to-Earth. In particular, it is unclear what the results of [11] tell one about the "classical" Lê numbers of a function on an affine space. We make these translations in Section 3 of this paper.

What we show is that the Lê numbers are actually the ranks of the free Abelian groups - the Lê modules - appearing in a complex whose cohomology is the integral cohomology of the Milnor fiber. Moreover, the Milnor monodromy acts naturally on this Lê module complex.

In Section 4, we prove that the traces of the monodromy actions on the Lê module complex depend solely on the topology/geometry of the critical locus of $f$.

Received by the editors October 12, 2004 and, in revised form, February 13, 2005.

2000 Mathematics Subject Classification. Primary 32B15, 32C35, 32C18, 32B10.

Key words and phrases. Lê modules, Lê numbers, Lê cycles, monodromy, Milnor fiber, vanishing cycles.

(C)2006 American Mathematical Society Reverts to public domain 28 years from publication 
This places some interesting restrictions on the characteristic polynomials of the Milnor monodromy action on the cohomology of the Milnor fiber.

\section{Quick REVIEW of LÊ NUMBERS}

Let $\mathcal{U}$ be an open neighborhood of the origin in $\mathbb{C}^{n+1}$ and let $f:(\mathcal{U}, \mathbf{0}) \rightarrow(\mathbb{C}, 0)$ be complex analytic. Let $s:=\operatorname{dim}_{0} \Sigma f$. We will assume throughout this paper that $\mathcal{U}$ is small enough so that $\Sigma f \subseteq V(f)$.

If $s=0$, then the Milnor number $\mu_{f}(\mathbf{0})$ of $f$ at the origin is an extremely important piece of numerical data related to the topology of the hypersurface $V(f)$. In particular, the reduced cohomology $\widetilde{H}^{*}()$ of the Milnor fiber $F_{f, \mathbf{0}}$ of $f$ at the origin is zero in all degrees, except possibly in degree $n$, and $\widetilde{H}^{n}\left(F_{f, \mathbf{0}}\right) \cong \mathbb{Z}^{\mu_{f}(\mathbf{0})}$.

Fix a set of linear coordinates $\mathbf{z}:=\left(z_{0}, \ldots, z_{n}\right)$ for $\mathcal{U}$. The Lê numbers of $f$, $\lambda_{f, \mathbf{z}}^{*}(\mathbf{0})$, with respect to $\mathbf{z}$ are a generalization of the Milnor number to the case where $s>0$. See [10. We shall not recall the definition of the Lê numbers here, but we will recall some of their properties.

The Lê numbers are defined algebraically, and their existence requires that the coordinates $\mathbf{z}$ are chosen generically in a fairly weak sense. If $\mathbf{z}$ is generic enough so that $\lambda_{f, \mathbf{z}}^{*}(\mathbf{0})$ exists, then $\lambda_{f, \mathbf{z}}^{*}(\mathbf{p})$ exists for all $\mathbf{p} \in V(f)$ near $\mathbf{0}$. It is only the choice of the coordinates $\left(z_{0}, \ldots, z_{s-1}\right)$ that matter in calculating the Lê numbers near the origin (recall that $s$ is calculated at the origin).

The $i$-dimensional Lê number at $\mathbf{0}, \lambda_{f, \mathbf{z}}^{i}(\mathbf{0})$, is possibly non-zero only for those $i$ such that $0 \leq i \leq s$. If $s=0$, the only conceivably non-zero Lê number is $\lambda_{f, \mathbf{z}}^{0}(\mathbf{0})$, which equals the Milnor number $\mu_{f}(\mathbf{0})$.

If $\mathcal{S}$ is a stratification of $V(f)$ which contains $V(f)-\Sigma f$ and such that all of the Lê numbers $\lambda_{f, \mathbf{z}}^{*}(\mathbf{p})$ remain constant as $\mathbf{p}$ varies along a stratum, then $\mathcal{S}$ is a good stratification, i.e., a stratification such that, for all $S \in \mathcal{S}$, the pair $(\mathcal{U}-V(f), S)$ satisfies the $a_{f}$ condition.

If one fixes a good stratification $\mathcal{S}$ of $V(f)$ in a neighborhood of the origin, the coordinates $z_{0}, \ldots, z_{s-1}$ are prepolar at the origin with respect to $\mathcal{S}$ provided that, for all $j$ such that $0 \leq j \leq s-1, V\left(z_{j}\right)$ transversely intersects all of the strata of $\mathcal{S} \cap V\left(z_{0}, \ldots, z_{j-1}\right)$ in a neighborhood of the origin except, perhaps, at the origin itself. Prepolar coordinates are generic. If $z_{0}, \ldots, z_{s-1}$ are prepolar at the origin with respect to $\mathcal{S}$, then the coordinates are prepolar with respect to $\mathcal{S}$ at all points near the origin. If the coordinates $z_{0}, \ldots, z_{s-1}$ are prepolar at the origin with respect to some good stratification, then there is a chain complex

$$
0 \rightarrow \mathbb{C}^{\lambda_{f}^{n}} \rightarrow \mathbb{C}^{\lambda_{f}^{n-1}} \rightarrow \cdots \rightarrow \mathbb{C}^{\lambda_{f}^{1}} \rightarrow \mathbb{C}^{\lambda_{f}^{0}} \rightarrow 0
$$

with cohomology isomorphic to $\widetilde{H}^{*}\left(F_{f, \mathbf{0}} ; \mathbb{C}\right)$, where we have written $\lambda_{f}^{i}$ for $\lambda_{f, \mathbf{z}}^{i}(\mathbf{0})$, and the $\mathbb{C}^{\lambda_{f}^{i}}$ term stands in degree $n-i$ (this complex is mistakenly reversed in [10]).

\section{More ReCEnt RESUlts}

In [11, the results that we proved were so general that it may be difficult to see what they say about the Lê numbers. This section will clarify the matter. Despite the fact that the results here follow quickly from the results of [1], we state them as theorems, since we have never before presented them as we do below. 
We continue to let $\mathbf{z}=\left(z_{0}, \ldots, z_{n}\right)$ be a set of linear coordinates for $\mathcal{U}$, and we will simplify our notation by also writing $z_{0}, \ldots, z_{n}$ when we restrict these coordinate functions to various subspaces.

Nearby and vanishing cycles. We need to take nearby and vanishing cycles with respect to $f$ and our various coordinates functions. Let us first recall some basic facts about these functors.

Suppose that $h: X \rightarrow \mathbb{C}$ is an analytic function. Fix a reasonably nice base ring (e.g., a P.I.D. or a field) $R$. Let $D_{c}^{b}(X)$ denote the derived category of bounded, constructible complexes of sheaves of $R$-modules on $X$.

Let $j_{h}^{*}$ denote the restriction functor from $D_{c}^{b}(X)$ to $D_{c}^{b}(V(h))$, which restricts a complex on $X$ to $V(h)$. We wish to discuss two other functors, $\psi_{h}$ and $\phi_{h}$, the nearby cycles along $h$ and the vanishing cycles along $h$, respectively, from $D_{c}^{b}(X)$ to $D_{c}^{b}(V(h))$.

If $\mathbf{A}^{\bullet} \in D_{c}^{b}(X)$, then the stalk cohomology of $\psi_{h} \mathbf{A}^{\bullet}$ at a point $x \in V(h)$ is isomorphic to the hypercohomology of the Milnor fiber of $h$ at $x$ with coefficients in $\mathbf{A}^{\bullet}$, i.e., $H^{i}\left(\psi_{h} \mathbf{A}^{\bullet}\right)_{x} \cong \mathbb{H}^{i}\left(F_{h, x} ; \mathbf{A}^{\bullet}\right)$. In particular, when applied to the constant sheaf, we obtain $H^{i}\left(\psi_{h} R_{X}^{\bullet}\right)_{x} \cong H^{i}\left(F_{h, x} ; R\right)$.

If $\mathbf{A}^{\bullet} \in D_{c}^{b}(X)$, then the stalk cohomology of $\phi_{h} \mathbf{A}^{\bullet}$ at a point $x \in V(h)$ is isomorphic to the shifted relative hypercohomology of the pair consisting of an open ball in $X$ and the Milnor fiber of $h$ at $x$ with coefficients in $\mathbf{A}^{\bullet}$, i.e., $H^{i}\left(\phi_{h} \mathbf{A}^{\bullet}\right)_{x} \cong$ $\mathbb{H}^{i+1}\left(\stackrel{\circ}{B}_{\epsilon}(x) \cap X, F_{h, x} ; \mathbf{A}^{\bullet}\right)$. In particular, when applied to the constant sheaf, we obtain $H^{i}\left(\phi_{h} R_{X}^{\bullet}\right)_{x} \cong \widetilde{H}^{i}\left(F_{h, x} ; R\right)$, where $\widetilde{H}$ denotes reduced cohomology.

There are natural maps $\operatorname{comp}_{h}: j_{h}^{*} \rightarrow \psi_{h}$ and $\operatorname{can}_{h}: \psi_{h} \rightarrow \phi_{h}$; these are the comparison map and canonical map, respectively. For all $\mathbf{A}^{\bullet} \in D_{c}^{b}(X)$, there is the natural distinguished triangle (which we write in-line):

$$
j_{h}^{*} \mathbf{A}^{\bullet} \stackrel{\operatorname{comp}_{h} \mathbf{A}^{\bullet}}{\longrightarrow} \psi_{h} \mathbf{A}^{\bullet} \stackrel{\operatorname{can}_{h} \mathbf{A}^{\bullet}}{\longrightarrow} \phi_{h} \mathbf{A}^{\bullet} \stackrel{[1]}{\longrightarrow} \text {. }
$$

The shifted functors $\psi_{h}[-1]:=\psi_{h} \circ[-1]$ and $\phi_{h}[-1]:=\phi_{h} \circ[-1]$ behave more nicely, in a functorial sense, than do the unshifted functors; in particular, $\psi_{h}[-1]$ and $\phi_{h}[-1]$ take perverse sheaves on $X$ to perverse sheaves on $V(h)$. For this reason, we always include the shifts, and we write the canonical distinguished triangle above as

$$
j_{h}^{*}[-1] \mathbf{A}^{\bullet} \stackrel{\mathrm{comp}_{h} \mathbf{A}^{\bullet}}{\longrightarrow} \psi_{h}[-1] \mathbf{A}^{\bullet} \stackrel{\operatorname{can}_{h} \mathbf{A}^{\bullet}}{\longrightarrow} \phi_{h}[-1] \mathbf{A}^{\bullet} \stackrel{[1]}{\longrightarrow},
$$

where, of course, $j_{h}^{*}[-1]:=j_{h}^{*} \circ[-1]$.

There are natural Milnor monodromy automorphisms $T_{h}: \psi_{h}[-1] \rightarrow \psi_{h}[-1]$ and $\widetilde{T}_{h}: \phi_{h}[-1] \rightarrow \phi_{h}[-1]$ such that, for all $\mathbf{A}^{\bullet} \in D_{c}^{b}(X)$, for all $x \in V(h)$, $T_{h} \mathbf{A}^{\bullet}$ and $\widetilde{T}_{h} \mathbf{A}^{\bullet}$ induce the Milnor monodromy actions on both $\mathbb{H}^{*}\left(F_{h, x} ; \mathbf{A}^{\bullet}\right)$ and $\mathbb{H}^{*}\left(\stackrel{\circ}{B}_{\epsilon}(x) \cap X, F_{h, x} ; \mathbf{A}^{\bullet}\right)$, respectively. The triple (id, $\left.T_{h} \mathbf{A}^{\bullet}, \widetilde{T}_{h} \mathbf{A}^{\bullet}\right)$ acts on the triangle $(\dagger)$ commutatively.

Now that we have reviewed nearby and vanishing cycles, we will continue to describe some of our more recent results.

Following Definition 5.1 of [1], but simplifying our notation for our current setting, we make the following definition. 
Definition 3.1. For all $j$ such that $0 \leq j \leq n$, define $\Phi_{f, \mathbf{z}}^{j}$ to be the complex of sheaves of $\mathbb{Z}$-modules given by iterating the vanishing and nearby cycles

$$
\Phi_{f, \mathbf{z}}^{j}:=\phi_{z_{j}}[-1] \psi_{z_{j-1}}[-1] \ldots \psi_{z_{0}}[-1] \phi_{f}[-1] \mathbb{Z}_{\mathcal{U}}[n+1]
$$

where, when $j=0$, we mean that $\Phi_{f, \mathbf{z}}^{0}:=\phi_{z_{0}}[-1] \phi_{f}[-1] \mathbb{Z}_{\mathcal{U}}^{\bullet}[n+1]$.

We say that the coordinates $\mathbf{z}$ are $\phi_{f}[-1] \mathbb{Z}_{\mathcal{U}}[n+1]$-isolating at $\mathbf{0}$ if and only if, for all $j$ such that $0 \leq j \leq s-1, \operatorname{dim}_{\mathbf{0}} \operatorname{supp}\left(\Phi_{f, \mathbf{z}}^{j}\right) \leq 0$.

Remark 3.2. Iterated vanishing and nearby cycles, such as we use above, seem to have first appeared in the literature in Sabbah's paper [12.

As $\mathbb{Z}_{\mathcal{U}}[n+1]$ is a perverse sheaf, and as shifted nearby and vanishing cycles take perverse sheaves to perverse sheaves, we see that each $\Phi_{f, \mathbf{z}}^{j}$ is a perverse sheaf. If the coordinates $\mathbf{z}$ are $\phi_{f}[-1] \mathbb{Z}_{\mathcal{U}}[n+1]$-isolating, then each $\Phi_{f, \mathbf{z}}^{j}$ is a perverse sheaf with the origin as an isolated point of its support; this implies that the stalk cohomology at the origin of each $\Phi_{f, \mathbf{z}}^{j}$ is concentrated in degree 0 .

Hence, we make the following definition:

Definition 3.3. If the coordinates $\mathbf{z}$ are $\phi_{f}[-1] \mathbb{Z}_{\mathcal{U}}^{\bullet}[n+1]$-isolating at the origin, then we define the $j$-dimensional Lê module of $f$ with respect to $\mathbf{z}$ at the origin to be

$$
M_{f, \mathbf{z}}^{j}:=H^{0}\left(\Phi_{f, \mathbf{z}}^{j}\right)_{\mathbf{0}} .
$$

The next theorem follows from results in [11. While we cannot reproduce, in a reasonable amount of space, the proofs from [11 that we need, we can easily describe the differentials $M_{f, \mathbf{z}}^{j} \stackrel{\partial_{j}}{\longrightarrow} M_{f, \mathbf{z}}^{j-1}$ which appear below.

Let

$$
\Psi_{f, \mathbf{z}}^{j-1}:=\psi_{z_{j-1}}[-1] \psi_{z_{j-2}}[-1] \ldots \psi_{z_{0}}[-1] \phi_{f}[-1] \mathbb{Z}_{\mathcal{U}}[n+1] .
$$

Then the canonical morphism in (†) yields a morphism $\Psi_{f, \mathbf{z}}^{j-1} \stackrel{\operatorname{can}_{j-1}}{\longrightarrow} \Phi_{f, \mathbf{z}}^{j-1}$. Applying the functor $\phi_{z_{j}}[-1]$, we obtain the morphism

$$
\Phi_{f, \mathbf{z}}^{j}=\phi_{z_{j}}[-1] \Psi_{f, \mathbf{z}}^{j-1} \stackrel{\phi_{z_{j}}[-1]\left(\operatorname{can}_{j-1}\right)}{\longrightarrow} \phi_{z_{j}}[-1] \Phi_{f, \mathbf{z}}^{j-1},
$$

and a corresponding morphism on stalk cohomology

$$
H^{0}\left(\Phi_{f, \mathbf{z}}^{j}\right)_{\mathbf{0}} \stackrel{\partial_{j}^{\prime}}{\longrightarrow} H^{0}\left(\phi_{z_{j}}[-1] \Phi_{f, \mathbf{z}}^{j-1}\right)_{\mathbf{0}} .
$$

Now, if the coordinates $\mathbf{z}$ are $\phi_{f}[-1] \mathbb{Z}_{\mathcal{U}}[n+1]$-isolating at the origin, then there exists a neighborhood $\mathcal{W}$ of the origin such that $\Phi_{f, \mathbf{z}}^{j-1}$ is zero when restricted to $\mathcal{W}-\{\mathbf{0}\}$ and, hence, $\mathbf{0}$ is not in the support of $\psi_{z_{j}}[-1] \Phi_{f, \mathbf{z}}^{j-1}$. Therefore, the long exact sequence on stalk cohomology given by the canonical distinguished triangle (†) yields an isomorphism $H^{0}\left(\phi_{z_{j}}[-1] \Phi_{f, \mathbf{z}}^{j-1}\right)_{0} \cong H^{0}\left(\Phi_{f, \mathbf{z}}^{j-1}\right)_{\mathbf{0}}$; by composing this isomorphism with the map $\partial_{j}^{\prime}$ above, we obtain a map $M_{f, \mathbf{z}}^{j} \stackrel{\partial_{j}}{\longrightarrow} M_{f, \mathbf{z}}^{j-1}$.

Theorem 3.4. Suppose that the coordinates $\mathbf{z}$ are $\phi_{f}[-1] \mathbb{Z}_{\mathcal{U}}[n+1]$-isolating at the origin. Then:

i) $M_{f, \mathbf{z}}^{j}=0$ for all $j>s$;

ii) if $s:=\operatorname{dim}_{0} \Sigma f \geq 1, M_{f, \mathbf{z}}^{s}=\psi_{z_{s-1}}[-1] \psi_{z_{s-2}}[-1] \ldots \psi_{z_{0}}[-1] \phi_{f}[-1] \mathbb{Z}_{\mathcal{U}}[n+1]$;

iii) for all $j, M_{f, \mathbf{z}}^{j}$ is free Abelian; 
iv) there is a complex of $\mathbb{Z}$-modules

$$
0 \stackrel{\partial_{s+1}}{\longrightarrow} M_{f, \mathbf{z}}^{s} \stackrel{\partial_{s}}{\longrightarrow} M_{f, \mathbf{z}}^{s-1} \stackrel{\partial_{s-1}}{\longrightarrow} \cdots \stackrel{\partial_{2}}{\longrightarrow} M_{f, \mathbf{z}}^{1} \stackrel{\partial_{1}}{\longrightarrow} M_{f, \mathbf{z}}^{0} \stackrel{\partial_{0}}{\longrightarrow} 0
$$

such that, for all $j$, the cohomology $\operatorname{ker} \partial_{j} / \operatorname{im} \partial_{j+1}$ is isomorphic to $\widetilde{H}^{n-j}\left(F_{f, \mathbf{0}} ; \mathbb{Z}\right)$;

v) the Milnor monodromy automorphism acts on the complex in iv) in a compatible fashion with the Milnor monodromy action on $\widetilde{H}^{*}\left(F_{f, \mathbf{0}} ; \mathbb{Z}\right)$;

vi) the induced Milnor monodromy in $\mathrm{v}$ ) acts on each $M_{f, \mathbf{z}}^{j}$ quasi-unipotently, i.e., the complex roots of the characteristic polynomials are all roots of unity.

Proof. We refer to Section 5 of [11] extensively. The complex $\mathbf{A}^{\bullet}$ of Section 5 of [1] will here be $\phi_{f}[-1] \mathbb{Z}_{\mathcal{U}}[n+1]$. The number $d$ of Section 5 of [11] will here be $s$. We will concentrate our attention at the origin.

Parts i) and ii) follow immediately from Corollary 5.15 of 11. Part iii) follows immediately from Theorem 5.23 and Remark 5.24 of [11. Part iv) follows immediately from Theorem 5.18 of [11. Part v) follows from the naturality of the monodromy action on the vanishing cycles.

We need to show part vi). Let $\widetilde{T}_{f}$ denote the monodromy automorphism on $\phi_{f}[-1] \mathbb{Z}_{\mathcal{U}}^{\bullet}[n+1]$. In a neighborhood of the origin, the Monodromy Theorem [2], [4], 6], 8] implies that there exist $a, b \in \mathbb{N}$ such that $\left(\mathrm{id}-\widetilde{T}_{f}^{a}\right)^{b}=0$ in the derived category; one applies the functors $\phi_{z_{j}}[-1] \psi_{z_{j-1}}[-1] \ldots \psi_{z_{0}}[-1]$ to this equality, and the conclusion follows immediately.

Definition 3.5. We refer to the complex appearing in Theorem 3.4 as the $L \hat{e}$ module complex of $f$ at $\mathbf{0}$ with respect to $\mathbf{z}$. We refer to the automorphism $\alpha_{j}$ on $M_{f, \mathbf{z}}^{j}$ which is induced by the Milnor monodromy as the $j$-dimensional L $\hat{e}$-Milnor monodromy (or, simply, LM monodromy).

We must still establish relationships between $\phi_{f}[-1] \mathbb{Z}_{\mathcal{U}}^{\bullet}[n+1]$-isolating coordinates and prepolar coordinates, and between the Lê numbers and the ranks of the Lê modules.

Let $\mathrm{Bl}_{J(f)} \mathcal{U} \stackrel{\pi}{\longrightarrow} \mathcal{U}$ denote the blow-up of $\mathcal{U}$ along the Jacobian ideal $J(f)$ of $f$. This blow-up naturally sits inside $\mathcal{U} \times \mathbb{P}^{n}$. Let $E$ denote the exceptional divisor of this blow-up. Below, $\pi_{*}$ denotes the proper push-forward of cycles.

Theorem 3.6. The following are equivalent:

a) The coordinates $\mathbf{z}$ are $\phi_{f}[-1] \mathbb{Z}_{\mathcal{U}}[n+1]$-isolating at the origin;

b) there exists an open neighborhood $\mathcal{W}$ of the origin inside $\mathcal{U}$ such that, for all $j$ such that $0 \leq j \leq s-1$, E properly intersects $\mathcal{W} \times\left(\mathbb{P}^{j} \times\{\mathbf{0}\}\right)$ inside $\mathcal{U} \times \mathbb{P}^{n}$ (i.e., the intersection is purely $j$-dimensional) and

$$
\operatorname{dim}_{\mathbf{0}}\left(V\left(z_{0}, \ldots, z_{j-1}\right) \cap \pi\left(E \cap\left(\mathcal{W} \times\left(\mathbb{P}^{j} \times\{\mathbf{0}\}\right)\right)\right) \leq 0\right.
$$

c) there exists an open neighborhood $\mathcal{W}$ of the origin inside $\mathcal{U}$ such that, for all $j$ such that $0 \leq j \leq n, E$ properly intersects $\mathcal{W} \times\left(\mathbb{P}^{j} \times\{\mathbf{0}\}\right)$ inside $\mathcal{U} \times \mathbb{P}^{n}$ and

$$
\operatorname{dim}_{\mathbf{0}}\left(V\left(z_{0}, \ldots, z_{j-1}\right) \cap \pi_{*}\left(E \cdot\left(\mathcal{W} \times\left(\mathbb{P}^{j} \times\{\mathbf{0}\}\right)\right)\right) \leq 0 .\right.
$$

If the equivalent conditions above hold, then, for all $j$ such that $0 \leq j \leq n$,

i) the germ of the $j$-dimensional Lê cycle $\Lambda_{f, \mathbf{z}}^{j}$ is defined and is equal to

$$
\pi_{*}\left(E \cdot\left(\mathcal{W} \times\left(\mathbb{P}^{j} \times\{\mathbf{0}\}\right)\right)\right) ;
$$


ii) the $j$-dimensional Lê number $\lambda_{f, \mathbf{z}}^{j}(\mathbf{0})$ is defined and is equal to the rank of the $j$-dimensional Lê module $M_{f, \mathbf{z}}^{j}$.

Proof. Except for the equivalence of b) to a) and c), this is Theorem 6.7 of [11, where $\mathbf{F}^{\bullet}:=\mathbb{Z}_{\mathcal{U}}^{\bullet}[n+1]$ and $\tilde{f}:=f$. The equivalence of b) to a) and c) follows at once from Theorem 5.10 of [11].

Remark 3.7. In fact, most of the results of [11] hold with coefficients in extremely general base rings $R$; in particular, they hold when $R$ is a finite field such as $\mathbb{Z} / p \mathbb{Z}$.

Thus, the more general form of Theorem 3.6 tells us that the coordinates $\mathbf{z}$ are $\phi_{f}[-1] \mathbb{Z}_{\mathcal{U}}^{\bullet}[n+1]$-isolating at the origin if and only if they are $\phi_{f}[-1] R_{\mathcal{U}}^{\bullet}[n+1]$ isolating at the origin, and when these equivalent conditions hold, there is a complex

$$
0 \rightarrow R^{\lambda_{f, \mathbf{z}}^{s}(\mathbf{0})} \rightarrow R^{\lambda_{f, \mathbf{z}}^{s-1}(\mathbf{0})} \rightarrow \cdots \rightarrow R^{\lambda_{f, \mathbf{z}}^{0}(\mathbf{0})} \rightarrow 0
$$

whose cohomology is isomorphic to $\widetilde{H}^{*}\left(F_{f, \mathbf{0}} ; R\right)$. In other words, the "Lê numbers with $R$ coefficients" remain the same.

Of course, by the Universal Coefficient Theorem,

$$
\widetilde{H}^{j}\left(F_{f, \mathbf{0}} ; R\right) \cong\left(\widetilde{H}^{j}\left(F_{f, \mathbf{0}} ; \mathbb{Z}\right) \otimes R\right) \oplus \operatorname{Tor}\left(\widetilde{H}^{j+1}\left(F_{f, \mathbf{0}} ; \mathbb{Z}\right), R\right) .
$$

Therefore, by varying $R$ through $\mathbb{Z}$ and through the fields $\mathbb{Z} / p \mathbb{Z}$, we find that not only do the Lê numbers put restrictions on the Betti numbers of $\widetilde{H}^{*}\left(F_{f, \mathbf{0}} ; \mathbb{Z}\right)$, they also place upper-bounds on the number of $p$-torsion direct summands of $\widetilde{H}^{*}\left(F_{f, \mathbf{0}} ; \mathbb{Z}\right)$.

Finally, below, we see why prepolar coordinates are important.

Theorem 3.8. Suppose the coordinates $\mathbf{z}$ are prepolar for $f$ at the origin. Then, the coordinates are $\phi_{f}[-1] \mathbb{Z}_{\mathcal{U}}[n+1]$-isolating at the origin, $f_{0}:=f_{\left.\right|_{V\left(z_{0}, \ldots, z_{s-1}\right)}}$ has an isolated critical point at the origin, and $\widetilde{H}^{n-s}\left(F_{f, \mathbf{0}} ; \mathbb{Z}\right) \cong$ ker $\partial_{s}$ injects into $\widetilde{H}^{n-s}\left(F_{f_{0}, 0} ; \mathbb{Z}\right)$ by a Milnor monodromy-invariant map.

Proof. That the coordinates $\mathbf{z}$ are $\phi_{f}[-1] \mathbb{Z}_{\mathcal{U}}[n+1]$-isolating is immediate from the second statement in Theorem 6.5 of [11]. Note that in our simple setting, the $a_{f, \mathbf{F}} \bullet$ partition referred to in 6.5 of [11] is simply a good partition. See Definition 4.7 of [11, use the fact that $\mathbf{F}^{\bullet}:=\mathbb{Z}_{\mathcal{U}}[n+1]$, and note that the only visible stratum is $\mathcal{U}-V(f)$.

The remaining statements follow from an inductive application of the main result of Lê in [7].

Remark 3.9. In the theorem above, only the statement about prepolar coordinates implying that the coordinates are $\phi_{f}[-1] \mathbb{Z}_{\mathcal{U}}[n+1]$-isolating is truly new. The comparison of the Milnor fiber of $f$ and of successive hyperplane slices of $f$ is a now-common technique, which originated with work of Hamm, Lê, and Teissier in 1973; see, for instance, [5], 7], and [15].

\section{Lê-Milnor traces}

In this section, we will show that the traces of the Lê-Milnor monodromies (Definition 3.5) can easily be described in terms of the topology of $\Sigma f$. We continue with $f, \mathbf{z}$, and $s$ as given in the previous sections. 
Definition 4.1. For all $j$ such that $0 \leq j \leq s$, we define the $j$-dimensional (complex) link of $\Sigma f$ at the origin with respect to $\mathbf{z}$ to be

$$
\mathbb{L}_{\Sigma f, \mathbf{z}}^{j}:=\stackrel{\circ}{B_{\epsilon}} \cap \Sigma f \cap V\left(z_{0}-a_{0}\right) \cap V\left(z_{1}-a_{1}\right) \cap \cdots \cap V\left(z_{s-j-1}-a_{s-j-1}\right),
$$

where $0 \ll\left|a_{0}\right| \ll\left|a_{1}\right| \ll \cdots \ll\left|a_{s-1}\right| \ll \epsilon \ll 1$. When $j=s$, we mean, of course, that $\mathbb{L}_{\Sigma f, \mathbf{z}}^{s}:=\stackrel{\circ}{B_{\epsilon}} \cap \Sigma f$. It is immediate that

$$
H^{k}\left(\mathbb{L}_{\Sigma f, \mathbf{z}}^{j} ; \mathbb{Z}\right) \cong H^{k}\left(\psi_{z_{s-j-1}} \psi_{z_{s-j-2}} \cdots \psi_{z_{0}} \mathbb{Z}_{\Sigma f}^{\bullet}\right)_{\mathbf{0}} .
$$

Note that we refer to $\mathbb{L}_{\Sigma f, \mathbf{z}}^{j}$ as the " $j$-dimensional link", even when the coordinates are such that $\mathbb{L}_{\Sigma f, \mathbf{z}}^{j}$ is not $j$-dimensional. However, generically, our terminology makes sense, since we have:

Proposition 4.2. If the coordinates $\mathbf{z}$ are $\phi_{f}[-1] \mathbb{Z}_{\mathcal{U}}[n+1]$-isolating at the origin, then, for all $j$ such that $0 \leq j \leq s, \mathbb{L}_{\Sigma f, \mathbf{z}}^{j}$ is $j$-dimensional.

Proof. If $\mathbf{z}$ are $\phi_{f}[-1] \mathbb{Z}_{\mathcal{U}}[n+1]$-isolating, then the Lê numbers are defined at the origin (Theorem [3.6ii). As the Lê numbers are defined, there is an equality of sets $\Sigma f=\bigcup_{i \leq s} \Lambda_{f, \mathbf{z}}^{i}$ and, for each $i, V\left(z_{0}, \ldots, z_{i-1}\right)$ properly intersects $\Lambda_{f, \mathbf{z}}^{i}$ at the origin. As $\Lambda_{f, \mathbf{z}}^{s}$ is non-empty (or, is not zero), the conclusion follows.

Note that it is definitely not true that isolating coordinates yield pure-dimensional $\mathbb{L}_{\Sigma f, \mathbf{z}}^{j}$.

So that we do not have to break the statement of the following theorem into two cases, we let $\mathbb{L}_{\Sigma f, \mathbf{z}}^{-1}:=\emptyset$.

Theorem 4.3. Suppose the coordinates $\mathbf{z}$ are $\phi_{f}[-1] \mathbb{Z}_{\mathcal{U}}[n+1]$-isolating at the origin. Then, for all $j$ such that $0 \leq j \leq s$, the trace of the $j$-dimensional Lê-Milnor monodromy $\alpha_{j}$ is

$$
(-1)^{n-j}\left(\chi\left(\mathbb{L}_{\Sigma f, \mathbf{z}}^{s-j-1}\right)-\chi\left(\mathbb{L}_{\Sigma f, \mathbf{z}}^{s-j}\right)\right) .
$$

Proof. Throughout this proof, if the Milnor monodromy acts on a complex $\mathbf{A}^{\bullet}$, we let $\mathcal{L}_{\mathbf{0}}\left\{\mathbf{A}^{\bullet}\right\}$ denote the Lefschetz number of the Milnor monodromy action on the stalk of $\mathbf{A}^{\bullet}$ at the origin. There is the fundamental distinguished triangle

$$
\mathbb{Z}_{V(f)}^{\bullet}[n] \rightarrow \psi_{f}[-1] \mathbb{Z}_{\mathcal{U}}^{\bullet}[n+1] \rightarrow \phi_{f}[-1] \mathbb{Z}_{\mathcal{U}}[n+1] \stackrel{[1]}{\longrightarrow} .
$$

Restricting to $\Sigma f$, we obtain

$$
\mathbb{Z}_{\Sigma f}^{\bullet}[n] \rightarrow\left(\psi_{f}[-1] \mathbb{Z}_{\mathcal{U}}^{\bullet}[n+1]\right)_{\left.\right|_{\Sigma f}} \rightarrow\left(\phi_{f}[-1] \mathbb{Z}_{\mathcal{U}}^{\bullet}[n+1]\right)_{\left.\right|_{\Sigma f}} \stackrel{[1]}{\longrightarrow} .
$$

Now, the support of $\phi_{f}[-1] \mathbb{Z}_{\mathcal{U}}^{\bullet}[n+1]$ is $\Sigma f$, and the monodromy action is the identity on $\mathbb{Z}_{\Sigma f}^{\bullet}[n]$. In addition, for all $\mathbf{p} \in \Sigma f, \mathrm{~A}^{\prime}$ Campo's result [1] implies that the Lefschetz number of the monodromy action on $\psi_{f}[-1] \mathbb{Z}_{\mathcal{U}}[n+1]$ at $\mathbf{p}$ is zero. It follows that, for all $j$ such that $0 \leq j \leq s-1$,

$$
\mathcal{L}_{0}\left\{\psi_{z_{j}}[-1] \ldots \psi_{z_{0}}[-1]\left(\left(\psi_{f}[-1] \mathbb{Z}_{\mathcal{U}}^{\bullet}[n+1]\right)_{\left.\right|_{\Sigma f}}\right)\right\}=0 .
$$


Therefore, for all $j$ such that $0 \leq j \leq s-1$,

$$
\begin{gathered}
\mathcal{L}_{\mathbf{0}}\left\{\psi_{z_{j}}[-1] \ldots \psi_{z_{0}}[-1] \phi_{f}[-1] \mathbb{Z}_{\mathcal{U}}^{\bullet}[n+1]\right\} \\
=-\chi_{\mathbf{0}}\left(\psi_{z_{j}}[-1] \ldots \psi_{z_{0}}[-1] \mathbb{Z}_{\Sigma f}^{\bullet}[n]\right)=(-1)^{n-j} \chi\left(\mathbb{L}_{\Sigma f, \mathbf{z}}^{s-j}\right),
\end{gathered}
$$

where $\chi_{\mathbf{0}}$ denotes the Euler characteristic of the stalk at $\mathbf{0}$.

Now, we find that, for all $j$ such that $1 \leq j \leq s$,

$$
\begin{gathered}
\mathcal{L}_{\mathbf{0}}\left\{\phi_{z_{j}}[-1] \psi_{z_{j-1}}[-1] \ldots \psi_{z_{0}}[-1] \phi_{f}[-1] \mathbb{Z}_{\mathcal{U}}[n+1]\right\} \\
=\mathcal{L}_{\mathbf{0}}\left\{\psi_{z_{j}}[-1] \ldots \psi_{z_{0}}[-1] \phi_{f}[-1] \mathbb{Z}_{\mathcal{U}}[n+1]\right\} \\
+\mathcal{L}_{\mathbf{0}}\left\{\psi_{z_{j-1}}[-1] \ldots \psi_{z_{0}}[-1] \phi_{f}[-1] \mathbb{Z}_{\mathcal{U}}[n+1]\right\} \\
=(-1)^{n-j} \chi\left(\mathbb{L}_{\Sigma f, \mathbf{z}}^{s-1}\right)+(-1)^{n-j-1} \chi\left(\mathbb{L}_{\Sigma f, \mathbf{z}}^{s-j}\right) \\
=(-1)^{n-j}\left(\chi\left(\mathbb{L}_{\Sigma f, \mathbf{z}}^{s-j-1}\right)-\chi\left(\mathbb{L}_{\Sigma f, \mathbf{z}}^{s-j}\right)\right) .
\end{gathered}
$$

When $j=0$, we use A'Campo's result again to obtain

$$
\begin{gathered}
\mathcal{L}_{\mathbf{0}}\left\{\phi_{z_{0}}[-1] \phi_{f}[-1] \mathbb{Z}_{\mathcal{U}}^{\bullet}[n+1]\right\} \\
=\mathcal{L}_{\mathbf{0}}\left\{\psi_{z_{0}}[-1] \phi_{f}[-1] \mathbb{Z}_{\mathcal{U}}^{\bullet}[n+1]\right\}+\mathcal{L}_{\mathbf{0}}\left\{\phi_{f}[-1] \mathbb{Z}_{\mathcal{U}}^{\bullet}[n+1]\right\} \\
=(-1)^{n} \chi\left(\mathbb{L}_{\Sigma f, \mathbf{z}}^{s-1}\right)+(-1)^{n+1}=(-1)^{n}\left(\chi\left(\mathbb{L}_{\Sigma f, \mathbf{z}}^{s-1}\right)-\chi\left(\mathbb{L}_{\Sigma f, \mathbf{z}}^{s}\right)\right) .
\end{gathered}
$$

Remark 4.4. Note that the alternating sum of the traces telescopes to yield the basic consequence of A'Campo's result from [1]: $\mathcal{L}_{\mathbf{0}}\left\{\phi_{f}[-1] \mathbb{Z}_{\mathcal{U}}[n+1]\right\}=(-1)^{n+1}$.

Thus, in a sense, what Theorem 3.4 and Theorem 4.3 tell us is that the Lê module complex refines the monodromy data given by the Monodromy Theorem and A'Campo's Lefschetz number theorem. More precisely, Theorem 3.4 and Theorem 4.3 use the fact that the Monodromy Theorem and A'Campo's theorem hold at points near the origin, and "compress" that data into a statement at the origin itself.

Results of this nature - that use the monodromy data at nearby points to deduce monodromy information at the origin - have certainly been studied by other authors; see, for instance, the work of Siersma in [14] and of Dimca in Chapter 6 of 3 .

Finally, related to Remark 3.7 we should mention that, since A'Campo's result remains true with arbitrary field coefficients, Theorem 4.3 is also true modulo $p$, when applied to $\alpha_{j}^{p}:=\alpha_{j} \otimes \mathrm{id}_{\mathbb{Z} / p \mathbb{Z}}$.

Corollary 4.5. Suppose that, at the origin, $\Sigma f$ is smooth and transversely intersected by $V\left(z_{0}, \ldots, z_{j}\right)$ for all $j$ such that $0 \leq j \leq s-1$. Suppose the coordinates $\mathbf{z}$ are $\phi_{f}[-1] \mathbb{Z}_{\mathcal{U}}^{\bullet}[n+1]$-isolating at the origin.

Then, the traces of the Lê-Milnor monodromies are all zero, except the s-dimensional one, which is $(-1)^{n-s-1}$. In particular, $\lambda_{f, \mathbf{z}}^{j}(\mathbf{0}) \neq 1$ for $j \neq s$.

Proof. The first statement follows immediately from Theorem 4.3 , since all of the links of $\Sigma f$ are contractible. The second statement follows from the fact that an automorphism of $\mathbb{Z}$ cannot have trace zero. 
Corollary 4.6. Suppose the coordinates $\mathbf{z}$ are $\phi_{f}[-1] \mathbb{Z} \bullet[n+1]$-isolating at the origin. Then, for all $j$ such that $0 \leq j \leq s$,

$$
\left|\chi\left(\mathbb{L}_{\Sigma f, \mathbf{z}}^{s-j-1}\right)-\chi\left(\mathbb{L}_{\Sigma f, \mathbf{z}}^{s-j}\right)\right| \leq \lambda_{f, \mathbf{z}}^{j}(\mathbf{0}),
$$

with equality holding for a given $j$ if and only if the characteristic polynomial of the $j$-dimensional Lê-Milnor monodromy is either $(t-1)^{\lambda_{f, \mathbf{z}}^{j}(\mathbf{0})}$ or $(t+1)^{\lambda_{f, \mathbf{z}}^{j}(\mathbf{0})}$.

Proof. This follows at once from Theorem 4.3 and the fact the complex eigenvalues of the LM monodromies are roots of unity (from Theorem 3.4 vi).

Corollary 4.7. Suppose that $\Sigma f$ is a set-theoretic local complete intersection at the origin. Suppose the coordinates $\mathbf{z}$ are $\phi_{f}[-1] \mathbb{Z}_{\mathcal{U}}^{\bullet}[n+1]$-isolating at the origin, and are also $\mathbb{Z}_{\Sigma f}^{\bullet}[s]$-isolating at the origin.

Then, all of the Lê-Milnor traces are either 0 or have the same sign as $(-1)^{n-s-1}$.

Proof. The proof of Theorem 4.3 shows that the $j$-dimensional LM trace is

$$
\begin{gathered}
-\chi_{0}\left\{\phi_{z_{j}}[-1] \psi_{z_{j-1}}[-1] \ldots \psi_{z_{0}}[-1] \mathbb{Z}_{\Sigma f}^{\bullet}[n]\right\} \\
=(-1)^{n-s-1} \chi_{\mathbf{0}}\left\{\phi_{z_{j}}[-1] \psi_{z_{j-1}}[-1] \ldots \psi_{z_{0}}[-1] \mathbb{Z}_{\Sigma f}^{\bullet}[s]\right\} .
\end{gathered}
$$

However, since $\Sigma f$ is a local complete intersection, $\mathbb{Z}_{\Sigma f}^{\bullet}[s]$ is a perverse sheaf. Thus,

$$
\mathbf{P}^{\bullet}:=\phi_{z_{j}}[-1] \psi_{z_{j-1}}[-1] \ldots \psi_{z_{0}}[-1] \mathbb{Z}_{\Sigma f}^{\bullet}[s]
$$

is a perverse sheaf. As the coordinates are $\mathbb{Z}_{\Sigma f}^{\bullet}[s]$-isolating, $\mathbf{P}^{\bullet}$ is a perverse sheaf which has the origin as an isolated point in its support; hence, the stalk cohomology of $\mathbf{P}^{\bullet}$ at the origin is concentrated in degree zero. The conclusion follows.

Remark 4.8. It is conceivable that $\phi_{f}[-1] \mathbb{Z}_{\mathcal{U}}[n+1]$-isolating coordinates are automatically $\mathbb{Z}_{\Sigma f}^{\bullet}[s]$-isolating, but we do not see a proof of this fact.

\section{EXAMPLES}

We will conclude by giving two examples.

Example 5.1. Suppose that, at the origin, $f$ has a smooth one-dimensional critical locus, $f_{\left.\right|_{V\left(z_{0}\right)}}$ has an isolated critical point, and $V\left(z_{0}\right)$ transversally intersects $\Sigma f$.

Then, $\lambda_{f, \mathbf{z}}^{1}(\mathbf{0})$ is simply equal to the Milnor number $\stackrel{\circ}{\mu}$ of a generic hyperplane slice of $f$ at a point on $\Sigma f$, sufficiently close to the origin. The 0-dimensional Lê number $\lambda_{f, \mathbf{z}}^{0}(\mathbf{0})$ is defined to be the intersection number $\left(\Gamma_{f, z_{0}}^{1} \cdot V\left(\frac{\partial f}{\partial z_{0}}\right)\right)_{\mathbf{0}}$, where $\Gamma_{f, z_{0}}^{1}$ is the relative polar curve of $f$ with respect to $z_{0}$.

Up to isomorphism, the Lê module complex of Theorem 3.4 becomes

$$
0 \rightarrow \mathbb{Z}^{\lambda_{f, \mathbf{z}}^{1}(\mathbf{0})} \stackrel{\partial_{1}}{\longrightarrow} \mathbb{Z}^{\lambda_{f, \mathbf{z}}^{0}(\mathbf{0})} \rightarrow 0
$$

where ker $\partial_{1} \cong \widetilde{H}^{n-1}\left(F_{f, \mathbf{0}}\right)$ and coker $\partial_{1} \cong \widetilde{H}^{n}\left(F_{f, \mathbf{0}}\right)$. Theorem 4.3 tells us that the 0 -dimensional LM monodromy has trace 0 , while the 1-dimensional LM monodromy has trace $(-1)^{n-2}=(-1)^{n}$.

In addition, by Remark 3.7 and Remark 4.4 the modulo $p$ version of the previous paragraph holds: for each prime $p$, we have a complex

$$
0 \rightarrow(\mathbb{Z} / p \mathbb{Z})^{\lambda_{f, \mathbf{z}}^{1}(\mathbf{0})} \stackrel{\partial_{1}^{p}}{\longrightarrow}(\mathbb{Z} / p \mathbb{Z})^{\lambda_{f, \mathbf{z}}^{0}(\mathbf{0})} \rightarrow 0
$$

where $\operatorname{ker} \partial_{1}^{p} \cong \widetilde{H}^{n-1}\left(F_{f, \mathbf{0}} ; \mathbb{Z} / p \mathbb{Z}\right)$ and coker $\partial_{1}^{p} \cong \widetilde{H}^{n}\left(F_{f, \mathbf{0}} ; \mathbb{Z} / p \mathbb{Z}\right)$. The modulo $p$ LM-monodromies $\alpha_{0}^{p}$ and $\alpha_{1}^{p}$ have traces equal to 0 and $(-1)^{n}$ in $\mathbb{Z} / p \mathbb{Z}$, respectively. 
If $\lambda_{f, \mathbf{z}}^{1}(\mathbf{0})=1$, then a well-known result of Siersma from 13 tells us that either $\lambda_{f, \mathbf{z}}^{0}(\mathbf{0})=0$ or $\partial_{1} \neq 0$ and, for all primes $p, \partial_{1}^{p} \neq 0$. It follows that there are two cases. In the first case, $\lambda_{f, \mathbf{z}}^{0}(\mathbf{0})=0, \widetilde{H}^{n-1}\left(F_{f, \mathbf{0}}\right) \cong \mathbb{Z}$, and $\widetilde{H}^{n}\left(F_{f, \mathbf{0}}\right)=0$. In the second case, $\lambda_{f, \mathbf{z}}^{0}(\mathbf{0}) \neq 0, \widetilde{H}^{n-1}\left(F_{f, \mathbf{0}}\right)=0$, and $\widetilde{H}^{n}\left(F_{f, \mathbf{0}}\right) \cong \mathbb{Z}^{\lambda_{f, \mathbf{z}}^{0}(\mathbf{0})-1}$.

What can one conclude if $\lambda_{f, \mathbf{z}}^{1}(\mathbf{0})=2$ ? A recent result of ours with Lê [9] tells us that, when $\operatorname{dim}_{\mathbf{0}} \Sigma f=1$, then the above statement - that either $\lambda_{f, \mathbf{z}}^{0}(\mathbf{0})=0$ or $\partial_{1} \neq 0$ and, for all primes $p, \partial_{1}^{p} \neq 0$-is always true.

As the LM-monodromies act invariantly on the kernel and image of $\partial_{1}$ (resp., $\left.\partial_{1}^{p}\right)$, and as trace $\left(\alpha_{1}\right)=(-1)^{n}$ (resp., $\operatorname{trace}\left(\alpha_{1}^{p}\right)=(-1)^{n}$ in $\mathbb{Z} / p \mathbb{Z}$ ), we cannot have that the rank (resp., dimension) of the kernel of $\partial_{1}$ (resp., $\partial_{1}^{p}$ ) is equal to 1.

Thus, it follows once again that there are two cases. In the first case, $\lambda_{f, \mathbf{z}}^{0}(\mathbf{0})=0$, $\widetilde{H}^{n-1}\left(F_{f, \mathbf{0}}\right) \cong \mathbb{Z}^{2}$, and $\widetilde{H}^{n}\left(F_{f, \mathbf{0}}\right)=0$. In the second case, $\lambda_{f, \mathbf{z}}^{0}(\mathbf{0}) \geq 2, \widetilde{H}^{n-1}\left(F_{f, \mathbf{0}}\right)$ $=0$, and $\widetilde{H}^{n}\left(F_{f, \mathbf{0}}\right) \cong \mathbb{Z}^{\lambda_{f, \mathbf{z}}^{0}(\mathbf{0})-2}$

Example 5.2. Let us look at an example in which the critical locus has more interesting topology. Suppose that, at the origin, $f$ has a critical locus which is the 2-dimensional cone $V\left(x^{2}+y^{2}+z^{2}, w_{1}, \ldots, w_{n-2}\right)$, where our coordinates on affine space are $\mathbf{z}:=\left(x, y, z, w_{1}, \ldots, w_{n-2}\right)$. We shall see that the topology of $\Sigma f$ forces the lower-dimensional Lê numbers to be non-zero, and puts other constraints on the cohomology and monodromy of the Milnor fiber of $f$ at the origin.

We denote homotopy-equivalence by $\simeq$. Of course, $\mathbb{L}_{\Sigma f, \mathbf{z}}^{2} \simeq$ point. The 1dimensional link, $\mathbb{L}_{\Sigma f, \mathbf{z}}^{1}$, is isomorphic to the Milnor fiber of $y^{2}+z^{2}$ in $\mathbb{C}^{2}$ and, hence, $\mathbb{L}_{\Sigma f, \mathbf{z}}^{1} \simeq S^{1}$. One easily finds that $\mathbb{L}_{\Sigma f, \mathbf{z}}^{0} \simeq 2$ points. Therefore, the respective Euler characteristics of $\mathbb{L}_{\Sigma f, \mathbf{z}}^{2}, \mathbb{L}_{\Sigma f, \mathbf{z}}^{1}$, and $\mathbb{L}_{\Sigma f, \mathbf{z}}^{0}$ are 1,0 , and 2 .

Assuming that the coordinates are $\phi_{f}[-1] \mathbb{Z}_{\mathcal{U}}[n+1]$-isolating at the origin, we find that $\operatorname{trace}\left(\alpha_{2}\right)=(-1)^{n-2}(0-2)=(-1)^{n-1} 2$, trace $\left(\alpha_{1}\right)=(-1)^{n-1}(2-0)=$ $(-1)^{n-1} 2$, and trace $\left(\alpha_{0}\right)=(-1)^{n-2}(0-1)=(-1)^{n-1}$. Furthermore, Corollary 3.3 of [9] tells us that $\partial_{2}$ cannot be the zero map.

Thus, we conclude from Corollary 4.6 that $\lambda_{f, \mathbf{z}}^{2}(\mathbf{0})$ and $\lambda_{f, \mathbf{z}}^{1}(\mathbf{0})$ must each be at least 2 , and $\lambda_{f, \mathbf{z}}^{0}(\mathbf{0}) \geq 1$. We also know that $\widetilde{H}^{n-2}\left(F_{f, \mathbf{0}}\right)$ is zero or isomorphic to $\mathbb{Z}$.

For special values of the Lê numbers, one can reach further conclusions. As in the previous example, let $\stackrel{\circ}{\mu}$ denote the Milnor number of a normal slice (i.e., the restriction to a generic codimension 2 linear subspace) of $f$ at a generic point on $\Sigma f$, sufficiently close to the origin. Then, $\lambda_{f, \mathbf{z}}^{2}(\mathbf{0}) \stackrel{\circ}{\mu} \cdot(\Sigma f \cdot V(x, y))_{\mathbf{0}}=2 \stackrel{\circ}{\mu}$, and so we see that $\lambda_{f, \mathbf{z}}^{2}(\mathbf{0})$ obtains its minimum value of 2 precisely when the Milnor number of a normal slice at a nearby generic point on $\Sigma f$ is 1 . This is the case, for instance, for $f_{1}:=w_{1}^{2}+\left(x^{2}+y^{2}+z^{2}\right)^{2}$.

Let us assume now that $\stackrel{\circ}{\mu}=1$ and, hence, $\lambda_{f, \mathbf{z}}^{2}(\mathbf{0})=2$. What more can we say about this case?

Since trace $\left(\alpha_{2}\right)=(-1)^{n-1} 2$, Corollary 4.6 tells us that the characteristic polynomial of $\alpha_{2}$, $\operatorname{char}_{\alpha_{2}}(t)$, would have to equal $\left(t-(-1)^{n-1}\right)^{2}$. If $\widetilde{H}^{n-2}\left(F_{f, \mathbf{0}}\right)=0$, then $\left(t-(-1)^{n-1}\right)^{2}$ must divide $\operatorname{char}_{\alpha_{1}}(t)$. If $\widetilde{H}^{n-2}\left(F_{f, \mathbf{0}}\right) \cong \mathbb{Z}$, then at least $\left(t-(-1)^{n-1}\right)$ must divide $\operatorname{char}_{\alpha_{1}}(t)$.

Now, if $\lambda_{f, \mathbf{z}}^{1}(\mathbf{0})=2$, then $\operatorname{char}_{\alpha_{1}}(t)$ must also be $\left(t-(-1)^{n-1}\right)^{2}$. If $\lambda_{f, \mathbf{z}}^{1}(\mathbf{0})=3$, we cannot be in the case where $\widetilde{H}^{n-2}\left(F_{f, \mathbf{0}}\right)=0$, i.e., the case where im $\partial_{2} \cong \mathbb{Z}^{2}$; 
for, otherwise, $\operatorname{trace}\left(\alpha_{2}\right)=(-1)^{n-1} 2$ and trace $\left(\alpha_{1}\right)=(-1)^{n-1} 2$ would imply that 0 would have to be the "third" eigenvalue of the isomorphism $\alpha_{1}$. Thus, if $\lambda_{f, \mathbf{z}}^{1}(\mathbf{0})=3$, then $\operatorname{im} \partial_{2} \cong \mathbb{Z}$, and the restriction of $\alpha_{1}$ to im $\partial_{2}$ has characteristic polynomial $\left(t-(-1)^{n-1}\right)$; it follows that $\operatorname{char}_{\alpha_{1}}(t)=\left(t-(-1)^{n-1}\right)\left(t^{2}-(-1)^{n-1} t+1\right)$, where it is important to note that $\left(t^{2}-(-1)^{n-1} t+1\right)$ is irreducible over $\mathbb{Z}$ (or $\mathbb{Q}$ ).

Therefore, when $\Sigma f$ is a 2 -dimensional cone and $\stackrel{\circ}{\mu}=1$, we have the following cases:

a) $\lambda_{f, \mathbf{z}}^{1}(\mathbf{0})=2, \widetilde{H}^{n-2}\left(F_{f, \mathbf{0}}\right)=0, \operatorname{rank} \widetilde{H}^{n-1}\left(F_{f, \mathbf{0}}\right)=0$, and $\widetilde{H}^{n}\left(F_{f, \mathbf{0}}\right) \cong \mathbb{Z}^{\lambda_{f, \mathbf{z}}^{0}(\mathbf{0})}$.

b) $\lambda_{f, \mathbf{z}}^{1}(\mathbf{0})=2, \widetilde{H}^{n-2}\left(F_{f, \mathbf{0}}\right) \cong \mathbb{Z}, \operatorname{rank} \widetilde{H}^{n-1}\left(F_{f, \mathbf{0}}\right)=0$, and $\operatorname{rank} \widetilde{H}^{n}\left(F_{f, \mathbf{0}}\right)=$ $\lambda_{f, \mathbf{z}}^{0}(\mathbf{0})-1$.

c) $\lambda_{f, \mathbf{z}}^{1}(\mathbf{0})=2, \widetilde{H}^{n-2}\left(F_{f, \mathbf{0}}\right) \cong \mathbb{Z}, \operatorname{rank} \widetilde{H}^{n-1}\left(F_{f, \mathbf{0}}\right)=1$, and $\widetilde{H}^{n}\left(F_{f, \mathbf{0}}\right) \cong$ $\mathbb{Z}_{f, \mathbf{z}}^{\lambda_{f}^{0}(\mathbf{0})}$.

d) $\lambda_{f, \mathbf{z}}^{1}(\mathbf{0})=3, \widetilde{H}^{n-2}\left(F_{f, \mathbf{0}}\right) \cong \mathbb{Z}, \operatorname{rank} \widetilde{H}^{n-1}\left(F_{f, \mathbf{0}}\right)=0$, and $\operatorname{rank} \widetilde{H}^{n}\left(F_{f, \mathbf{0}}\right)=$ $\lambda_{f, \mathbf{z}}^{0}(\mathbf{0})-2$.

e) $\lambda_{f, \mathbf{z}}^{1}(\mathbf{0})=3, \widetilde{H}^{n-2}\left(F_{f, \mathbf{0}}\right) \cong \mathbb{Z}, \operatorname{rank} \widetilde{H}^{n-1}\left(F_{f, \mathbf{0}}\right)=2$, the monodromy on the free part of $\widetilde{H}^{n-1}\left(F_{f, \mathbf{0}}\right)$ has characteristic polynomial $\left(t^{2}-(-1)^{n-1} t+1\right)$, and $\widetilde{H}^{n}\left(F_{f, \mathbf{0}}\right) \cong \mathbb{Z}^{\lambda_{f, \mathbf{z}}^{0}(\mathbf{0})}$.

f) $\lambda_{f, \mathbf{z}}^{1}(\mathbf{0}) \geq 4$.

As one should expect, we see that knowing the traces of the LM-monodromies, and knowing that the eigenvalues are roots of unity, yield strong results for small values of the Lê numbers.

\section{REFERENCES}

[1] A'Campo, N., Le nombre de Lefschetz d'une monodromie. Proc. Kon. Ned. Akad. Wet., Series A, 76:113-118, 1973. MR0320364 (47:8903)

[2] Clemens, C. H., Picard-Lefschetz theorem for families of non-singular algebraic varieties acquiring ordinary singularities. Trans. AMS, 136:93-108, 1969. MR0233814(38:2135)

[3] Dimca, A., Sheaves in Topology. Universitext. Springer-Verlag, 2004. MR2050072

[4] Grothendieck, A., Séminaire de Géométrie Algébrique (SGA VII-1), volume 288 of Lect. Notes. Math. Springer-Verlag, 1972. Résumé des premiers exposés de A. Grothendieck, rédigé par P. Deligne.

[5] Hamm, H. and Lê, D. T., Un théorème de Zariski du type de Lefschetz. Ann. Sci. Éc. Norm. Sup., 6 (series 4):317-366, 1973. MR0401755 (53:5582)

[6] Landman, A., On Picard-Lefschetz Transformation for algebraic manifolds acquiring general singularities. Trans. AMS, 181:89-126, 1973. MR0344248(49:8987)

[7] Lê, D. T., Calcul du Nombre de Cycles Evanouissants d'une Hypersurface Complexe. Ann. Inst. Fourier, Grenoble, 23:261-270, 1973. MR0330501(48:8838)

[8] Lê, D. T., The Geometry of the Monodromy Theorem. In C. P. Ramanujam - a tribute, Tata Institute, pages 157-173. Springer-Verlag, 1978. MR0541020 (80i:32031)

[9] Lê, D. T. and Massey, D., Hypersurface Singularities and the Swing. preprint, 2005.

[10] Massey, D., Lê Cycles and Hypersurface Singularities, volume 1615 of Lecture Notes in Math. Springer-Verlag, 1995. MR1441075 (98h:32061)

[11] Massey, D., Singularities and Enriched Cycles. Pacific J. Math., 215, no. 1:35-84, 2004. MR2060494 (2005f:32052)

[12] Sabbah, C., Proximité évanescente. Compos. Math., 62:283-328, $1987 . \quad$ MR0901394 (90a:32014)

[13] Siersma, D., Isolated line singularities. Proc. Symp. Pure Math., 35, part 2, Arcata Singularities Conf.:485-496, 1983. MR.0713274 (85d:32017) 
[14] Siersma, D., Variation mappings on singularities with a 1-dimensional critical locus. Topology, 30:445-469, 1991. MR1113689 (92e:32022)

[15] Teissier, B., Cycles évanescents, sections planes et conditions de Whitney. Astérisque, 78:285-362, 1973. MR0374482 (51:10682)

Department of Mathematics, Northeastern University, Boston, Massachusetts 02115 